Archives de sciences sociales des religions

160 | octobre-décembre 2012

Bulletin Bibliographique

\title{
Catholicisme en fusion
}

\section{Yvon Tranvouez}

\section{(2) OpenEdition}

Journals

Édition électronique

URL : http://journals.openedition.org/assr/24568

DOI : $10.4000 /$ assr. 24568

ISSN : $1777-5825$

Éditeur

Éditions de l'EHESS

Édition imprimée

Date de publication : 30 décembre 2012

Pagination : 91-100

ISSN : 0335-5985

\section{Référence électronique}

Yvon Tranvouez, "Catholicisme en fusion ", Archives de sciences sociales des religions [En ligne],

160 | octobre-décembre 2012, mis en ligne le 20 février 2016, consulté le 02 mai 2019. URL : http:// journals.openedition.org/assr/24568; DOI : 10.4000/assr.24568 


\title{
Catholicisme en tensions
}

\author{
À propos de : \\ Béraud Céline, Gugelot Frédéric, SaINT-Martin Isabelle (dir.), Catholi- \\ cisme en tensions, Paris, Éditions de l'EHESS, 2012, 326 p. Préface \\ de Danièle Hervieu-Léger.
}

\section{Yvon Tranvouez \\ Catholicisme en fusion}

En couverture, une belle photographie en contre-plongée de l'intérieur de la Sagrada Familia de Gaudi, l'un des monuments emblématiques du traitement moderne du religieux, illustrant parfaitement la célèbre formule de Michel de Certeau sur le passage du catholicisme du « corps » organisé au « corpus » disséminé. Le volume est dirigé par un trio représentatif de la nouvelle génération qui arrive aux commandes de la sociologie des religions ou de l'histoire religieuse du temps présent. La précédente donne sa bénédiction en ouverture (Danièle Hervieu-Léger) et en clôture (Jean-Paul Willaime). La suivante, encore peu connue du public, assure l'essentiel des vingt-et-une contributions, ce qui laisse augurer un salubre renouvellement des thèmes ou des approches. On pourrait craindre, évidemment, de se trouver en présence de l'un de ces ouvrages collectifs qui se multiplient depuis qu'une gestion étroitement comptable de la recherche universitaire régit les carrières et que la bibliométrie envahissante suscite des productions dispersées à l'identité mal assurée et au contenu inégal. Rien de tel ici, heureusement, et le lecteur est vite rassuré. Il y a une réelle cohérence dans ce regroupement d'études portant sur des pays occidentaux de tradition catholique : France, surtout, mais aussi Espagne, Italie et Québec. On voit également passer, ponctuellement, l'Allemagne et la Belgique. Une seule échappée inattendue, en Amérique latine (Argentine et Uruguay). La question générale est, comme le résume Jean-Paul Willaime, celle de «la capacité du catholicisme à se repenser et se repositionner comme une sous-culture minoritaire dans un environnement pluraliste, alors qu'il a longtemps constitué un cadre culturel englobant des sociétés étudiées" (p. 310). La réponse ne vient pas d'un panorama général mais d'une observation éclatée, délibérément plus attentive à ce qui se pratique à la base qu'à ce qui se dit au sommet de l'institution. Il s'agit de mettre en évidence un certain nombre de points critiques, révélateurs de la situation du catholicisme, au début du $\mathrm{XXI}^{\mathrm{e}}$ siècle, dans ce que l'on appelle généralement la postmodernité et que Jean-Paul Willaime préfère désigner comme ultramodernité (le logiciel 
Word souligne toujours le mot en rouge, signe que son auteur n'a pas encore réussi à en imposer l'usage).

L'ouvrage s'ouvre par un suggestif «état des lieux » en France, Espagne, Italie et Québec. On aurait pu imaginer une comparaison systématique à partir d'indices homogènes, mais c'est un autre parti qui a été pris : identifier les problèmes les plus significatifs dans chaque pays, ce qui permet de mesurer le poids des héritages nationaux. Philippe Portier analyse la pluralisation du paysage catholique en France et sa délicate gestion par la hiérarchie ecclésiastique. Alfonso Pérez-Agote montre la rapidité de la sécularisation de la société espagnole. Luca Diotallevi souligne la capacité d'influence que conserve le catholicisme italien dans une société devenue polyarchique. Solange Lefebvre pointe les contradictions des catholiques québécois tiraillés entre un modèle de laïcité à la française, soucieux de neutralisation religieuse de la sphère publique, et un modèle de sécularisation à l'anglo-saxonne, attentif aux droits des individus et des communautés.

Les études spécifiques qui suivent, distribuées en trois parties («clergé, pratiques, croyances »; " corps, genre, intimité »; " culture et catholicisme ») mettent en évidence un certain nombre de points de tension entre habitus catholique et pratiques modernes. Successivement, entre prêtres et diacres (Jean-René Bertrand) ; entre service de l'institution et épanouissement personnel chez les religieux (Brigitte Bleuzen); entre gestion hiérarchique et revendications démocratiques dans les synodes (Nicolas de Bremond d'Ars) ; entre logique religieuse et logique économique dans l'hôtellerie monastique (Isabelle Jonveaux) ; entre normes cléricales et usages populaires dans le culte des saints (Bruno Duriez); entre prescriptions institutionnelles et attentes personnelles dans la transmission des croyances (Elena Zapponi) comme dans la célébration des funérailles (Guénolé Labéy-Guimard); entre loi naturelle et droit de l'individu dans la gestion du corps (Denis Pelletier, Isacco Turina) et l'affirmation homosexuelle (David Koussens, Martine Gross) ; entre exclusivité masculine et prétentions féminines au sacerdoce (Sabine Rousseau) ou au service d'autel (Céline Béraud); entre image convenue et image décalée du prêtre dans le roman policier (Frédéric Gugelot) ; entre contrôle ecclésial et liberté de l'artiste dans le traitement des thèmes religieux par l'art contemporain (Isabelle Saint-Martin) ; entre respect et dérision du catholicisme dans la société française, au témoignage de René Rémond (Charles Mercier) ; entre appropriation religieuse et libre-service culturel de la Bible (Pierre Lassave). On excusera cet inventaire, qui s'imposait pour donner une idée de la richesse et de la diversité des contributions.

Ceci étant lu, trois leçons générales se dégagent. La première est que les tensions évoquées sont à la fois extérieures et intérieures. L'Église catholique se trouve en décalage, voire en conflit, avec la société actuelle, mais son problème ne se réduit pas à la détermination d'une ligne intransigeante ou de solutions de compromis avec un monde vis-à-vis duquel elle serait en quelque sorte en face 
à face. La problématique de "l'ouverture au monde », développée par le concile Vatican II, est dépassée en ce sens qu'il n'est plus possible de ne pas voir que les exigences modernes se manifestent au sein même de l'institution catholique. Cela était évidemment déjà le cas dans les années 1950 ou 1960, mais le bloc catholique semblait encore assez cohérent pour canaliser les forces centrifuges. Aujourd'hui, comme le souligne Céline Béraud (p. 246), la tension entre l'Église et le monde se double d'une tension ecclésiologique interne.

La deuxième leçon est que les lignes de front se sont déplacées. Les débats sur la famille et la bioéthique sont désormais les plus vifs. Battant en retraite, l'Église catholique doit donc défendre des positions qu'elle pensait assurées. Voici quarante ans, elle avait concédé à ses fidèles le pluralisme politique : la voilà aujourd'hui sommée de leur reconnaître le pluralisme des mœurs. Plus redoutable encore, peut-être, l'offensive menée, au nom du droit des femmes à l'égalité, contre le fonctionnement d'une institution dont les tâches décisives sont réservées aux hommes : que l'on réclame la possibilité d'ordonner des femmes prêtres ou que l'on demande l'indistinction des genres dans le service liturgique, on touche au sacré. Il est significatif qu'une mère de famille, dont les deux fillettes avaient été exclues du service de l'autel, ait cru pouvoir saisir la Halde (cas cité par Céline Béraud, p. 252). Le recrutement exclusivement masculin du clergé catholique pourrait aussi être lu comme une forme de discrimination à l'embauche, et la question a déjà été soulevée en ces termes en Angleterre. On voit comment la société entend contraindre l'institution catholique à se soumettre à ses propres exigences : les «droits de l'homme » font l'effet d'un sacré moderne auquel les normes religieuses sont priées de se conformer. Sans doute ceci n'est-il pas absolument nouveau. On se souvient qu'en 1790, déjà, l'Assemblée nationale avait prétendu contraindre les individus à ne pas aliéner leur liberté, et supprimé, de la secousse, tous les ordres religieux, au motif que leurs membres renonçaient à leur libre arbitre en se conformant à une règle. Et plus récemment, en 1995, le rapport parlementaire Gest-Guyard sur les sectes n'a évité de soulever des questions embarrassantes sur la vie monastique ou les communautés charismatiques, qu'en excluant a priori de son observation les organisations relevant des Églises.

La troisième leçon, qui amène précisément à voir la nouveauté de la situation actuelle, tient à ce que la plupart des tensions évoquées dans ce livre concernent le rapport du catholicisme à la société civile. Autrefois l'Église s'affrontait à l'État : les camps étaient tranchés, les positions balisées. Mais aujourd'hui, comme le souligne Denis Pelletier, l'État lui-même est en crise : sphère religieuse et sphère politique se trouvent confrontées à « une déstabilisation commune de leurs catégories fondatrices» (p. 189). L'institution catholique doit donc faire avec l'air du temps. Ses difficultés tiennent peut-être moins à la diminution de ses effectifs qu'au fait que ceux qui lui restent «fidèles » n'en font qu'à leur tête, comme tout le monde. Du coup, les synodes diocésains étudiés par Nicolas de Bremond d'Ars sont un extraordinaire révélateur de l'évolution de la fonction épiscopale, 
obligée de donner dans la gouvernance : enfumage des débats dans une litanie de témoignages "vécus » et "émouvants ", réassurance collective par l'effusion des célébrations inaugurales et conclusives où le soleil est forcément au rendezvous (s'il pleut, il est "dans les cœurs ») et, au final, verrouillage des questions qui fâchent (célibat sacerdotal, divorcés remariés, etc.) - entendues sans doute, écoutées peut-être, mais écartées assurément.

La plupart des contributions émanant de sociologues, on ne s'étonnera pas que les approches soient résolument contemporaines. Les auteurs regardent ce qui se passe aujourd'hui, au lieu de se demander, comme le ferait spontanément un historien, ce que devient aujourd'hui ce qui existait hier. Exit donc le discours de la "perte ", qui ne prédispose certes pas à percevoir le nouveau et qui finit par tourner en rond. Ceci étant, ce sont précisément des historiens qui soulèvent une question irrésolue sur le processus et les étapes du changement. Charles Mercier se demande comment on passe du René Rémond optimiste de Vivre notre histoire (1976), ou encore serein de l'Histoire de la France religieuse (1992), à celui, pessimiste, du Christianisme en accusation (2000). Analysant la question de l'ordination des femmes, Sabine Rousseau évoque pertinemment (p. 229) une longue "décennie du silence »(1982-1994) entre les deux phases (1970-1982 et 1994-2006) de la revendication féministe. Entre la contestation des années 1960-1970 et la transgression des années 1990-2000, il y aurait, me semble-t-il, à étudier de près la défection silencieuse des années 1980. Et pour cela, selon le bon vieux conseil d'Augustin Thierry, « remuer les faits » plus que les idées.

Le livre s'achève par une brillante conclusion de Blandine Chélini-Pont, qui donne le tournis en invitant à considérer le catholicisme actuel comme « une spirale qui, selon l'ajustement de l'œil, mettrait au centre ce qui est le plus à l'extérieur ou le plus à l'extérieur ce qui est au centre. De la sorte, rien de périphérique, rien de central, rien d'annexe et rien de fixe, si ce n'est dans l'instant de la perspective " (p. 315). Sujet au strabisme divergent, le recenseur sent venir la migraine, mais il poursuit sa lecture : «[...] Il faut chercher les recompositions incessantes de cette immense matrice, qui sont comme une acceptation du décomposé, une adaptation à l'interstice et à la frontière floues. [...] Mécanique des catégories et des ordres, le catholicisme évolue, et de ses étirements naît un organisme plastique et arborescent qui s'élargit par des canaux improbables, loin des raideurs qui semblent accabler son ossature " (p. 315). Alors, tensions ou liquéfaction ? Catholicisme en fusion, serais-je tenté de penser, mais il ne faut pas décourager les candidats à l'épiscopat.

Yvon TRANVOUEZ

UBO-Université de Bretagne occidentale-Brest yvon.tranvouez@bbox.fr 